\title{
Uniform measure density condition and game regularity for tug-of-war games
}

\author{
JOONAS HEINO
}

Department of Mathematics and Statistics, University of Jyväskylä, P.O. Box 35, FI-40014 Jyväskylä, Finland. E-mail: joonas.heino@jyu.fi

We show that a uniform measure density condition implies game regularity for all $2<p<\infty$ in a stochastic game called "tug-of-war with noise". The proof utilizes suitable choices of strategies combined with estimates for the associated stopping times and density estimates for the sum of independent and identically distributed random vectors.

Keywords: density estimate for the sum of i.i.d. random vectors; game regularity; hitting probability; $p$-harmonic functions; $p$-regularity; stochastic games; uniform distribution in a ball; uniform measure density condition

\section{Introduction}

The profound connection between stochastic processes and classical linear partial differential equations has been pivotal. For example, this connection was made use of in [8,9] to establish regularity result for the second order equations in a non-divergence form. Recently, a connection between nonlinear infinity harmonic functions and tug-of-war games was discovered in [15]. Later in [16], the authors found a stochastic game related to $p$-harmonic functions. They proved among other things by using a game approach that in a game regular domain there exists a $p$-harmonic function extending continuously to the boundary with the given continuous boundary values. However, a problem asking if a regular boundary point for the $p$-Laplacian is necessarily game regular was left open.

We study a modified version of a "tug-of-war with noise" developed in [13] and also related to $p$-harmonic functions. First, the players choose a step length $\epsilon>0$ and a starting point $x_{0}$. Then, they toss a biased coin, and if they get heads (probability $\alpha$ ), the players play a "tug-of-war", that is, they toss a fair coin and the winner of the toss can move the game position to any point of the open ball centered at $x_{0}$ and of the radius $\epsilon$. If in the first toss, they get tails (probability $\beta$ ), the game point moves according to the uniform distribution in the open ball centered at $x_{0}$ and of the radius $\epsilon$. After the first move, the players play the same game from the new game position. The game ends, when the game position exits the game domain for the first time. In the end, Player 2 pays to Player 1 the amount given by the payoff function at the first point outside the domain. We consider this version of the game because the players do not affect the direction of the noise and hence, we can prove sharp enough estimates for the density of the noise.

We give a stochastic proof that a uniform measure density condition implies game regularity (Theorem 3.7). Roughly, a boundary point $y$ is game regular, if Player 1 has a strategy to end the game near $y$ with a probability close to one whenever the game starts near $y$ as well. A boundary 
point $y$ satisfies a measure density condition, if the Lebesgue measure of the complement of the game domain in the ball centered at $y$ is comparable to the Lebesgue measure of the whole ball. The proof of Theorem 3.7 utilizes a stochastic density estimate for the sum of independent and identically distributed random vectors (Lemma A.4). In addition, we use a "cylinder walk" framework together with a cancellation strategy for Player 1 to connect the stochastic estimates to the setting. We omit the case $p=2$, because in that case the process is merely a random walk and the result follows from the classical invariance principle.

Game theory has already given new insights to partial differential equations. For instance, the ideas emerging from nonlinear game theory have led to simpler as well as completely different proofs for PDEs (see, for example, [1] and [10]). In addition, a dynamic programming principle related to the game also arises from discretization schemes (see, for instance, [14]).

We expect the techniques developed in this paper to be useful for a larger class of partial differential equations as well. In addition, stochastic estimates on where the game spends time under cancellation strategies are likely to be important for further results.

This work is organized as follows. In Section 2, we describe the preliminaries needed in the paper. Then in Section 3, we show that the uniform measure density condition implies game regularity for all $2<p<\infty$. For brevity, we do not write down all the stochastic calculations needed in the section, but the calculations are in the Appendix.

\section{Preliminaries}

First, let us start by introducing the notation. We denote the standard Euclidean open ball by $B_{r}\left(x_{0}\right) \subset \mathbb{R}^{n}$,

$$
B_{r}\left(x_{0}\right)=\left\{z \in \mathbb{R}^{n}:\left|z-x_{0}\right|<r\right\} .
$$

Lebesgue measure is denoted by $|\cdot|$, and in addition, the notation $C_{n, p}$ means that the universal constant depends only on $n$ and $p$. Throughout the paper, we use the asymptotic notation $\mathcal{O}(\epsilon)$. For example, if a real-valued function $f$ satisfies the inequality $f(\epsilon) \leq \mathcal{O}(\epsilon)$, it means that there exists a constant $C>0$ such that $|f(\epsilon)| \leq C \epsilon$ for all $\epsilon>0$ small enough.

Let $2<p<\infty, \epsilon>0$ and dimension $n \geq 1$. Fix a bounded, non-empty and open set $\Omega \subset \mathbb{R}^{n}$. Next, we recall the two-player zero-sum-game called "tug-of-war with noise". First, choose a starting point $x_{0} \in \Omega$ for the game, and then, the players toss a biased coin with probabilities $\alpha$ and $\beta$. The probabilities depend on $n$ and $p$ by

$$
\alpha=\frac{p-2}{p+n}, \quad \beta=\frac{n+2}{p+n} .
$$

The players get heads with the probability $\alpha$, and in this case, they will toss a fair coin and the winner of the toss can move the game position to any point of the open ball $B_{\epsilon}\left(x_{0}\right)$. Tossing of a fair coin and the movement after the toss are the "tug-of-war" parts of the game. On the other hand, if they get tails, the next game position will be decided by the uniform distribution in the ball $B_{\epsilon}\left(x_{0}\right)$. A random movement is the "noise" part of the game. After the first move is decided, the players continue playing the same game from the new position. 
The game procedure yields a sequence of game positions $x_{0}, x_{1}, x_{2}, \ldots$, where every $x_{k}$ is a random variable. A history of a game up to step $k$ is a vector of the first $k+1$ game positions $x_{0}, \ldots, x_{k}$ and $k$ coin tosses $c_{1}, \ldots, c_{k}$, that is,

$$
h_{k}:=\left(x_{0},\left(c_{1}, x_{1}\right), \ldots,\left(c_{k}, x_{k}\right)\right) .
$$

In the above, $c_{j} \in \mathcal{C}:=\{0,1,2\}$, where 0 denotes that Player 1 wins, 1 that Player 2 wins and 2 that a random movement occurs.

To prescribe boundary values, let us denote a compact boundary strip of width $\epsilon$ by

$$
\Gamma_{\epsilon}:=\left\{z \in \mathbb{R}^{n} \backslash \Omega: \inf _{y \in \partial \Omega}|z-y| \leq \epsilon\right\} .
$$

The reason to use the boundary strip instead of just the boundary is that $B_{\epsilon}(x) \subset \Omega_{\epsilon}:=\Omega \cup \Gamma_{\epsilon}$ for all $x \in \Omega$. After the first time the game position is in $\Gamma_{\epsilon}$, the players do not move it anymore. For all $k \geq 0$, the history $h_{k}$ belongs to the space $H^{k}:=x_{0} \times\left(\mathcal{C}, \Omega_{\epsilon}\right)^{k}$ with $H^{0}:=x_{0}$. We denote the space of all game sequences by

$$
H^{\infty}:=\bigcup_{k \geq 0} H^{k}=x_{0} \times\left(\mathcal{C}, \Omega_{\epsilon}\right) \times\left(\mathcal{C}, \Omega_{\epsilon}\right) \times \cdots
$$

A strategy for Player 1 is a sequence of Borel measurable functions that give the next game position given the history of the game. To be more precise, a strategy for Player 1 is $S_{1}:=$ $\left(S_{1, k}\right)_{k=0}^{\infty}$ with

$$
S_{1, k}: H^{k} \rightarrow \mathbb{R}^{n}
$$

for all $k \geq 0$. For example, if Player 1 wins the $(k+1)$ th toss,

$$
S_{1, k}\left(x_{0},\left(c_{1}, x_{1}\right), \ldots,\left(c_{k}, x_{k}\right)\right)=x_{k+1} \in B_{\epsilon}\left(x_{k}\right)
$$

for all $h_{k} \in H^{k}$. Similarly Player 2 deploys a strategy $S_{2}$.

We denote the first hitting time to the set $\Gamma_{\epsilon}$ by

$$
\tau:=\tau(\omega)=\inf \left\{k: x_{k} \in \Gamma_{\epsilon}, k=0,1,2, \ldots\right\} .
$$

The game process is a discrete time adapted process with respect to the filtration $\mathcal{F}_{0}:=\sigma\left(x_{0}\right)$ and

$$
\mathcal{F}_{k}:=\sigma\left(x_{0},\left(c_{1}, x_{1}\right), \ldots,\left(c_{k}, x_{k}\right)\right) \quad \text { for } k \geq 1,
$$

so $\tau$ is a stopping time. The game ends at the random time $\tau$, and the payoff is $F\left(x_{\tau}\right)$, where $F: \Gamma_{\epsilon} \rightarrow \mathbb{R}$ is a fixed, bounded and Borel measurable payoff function. In the end, Player 2 pays the amount $F\left(x_{\tau}\right)$ to Player 1 .

To establish a unique probability measure, we need to know a starting point $x_{0}$ and strategies $S_{1}$ and $S_{2}$. Then, the probability measure $\mathbb{P}_{S_{1}, S_{2}}^{x_{0}}$ on the natural product $\sigma$-algebra is built by 
applying Kolmogorov's extension theorem to the family of transition densities

$$
\begin{aligned}
& \pi_{S_{1}, S_{2}}\left(x_{0},\left(c_{1}, x_{1}\right), \ldots,\left(c_{k}, x_{k}\right),(C, A)\right) \\
& =\frac{\alpha}{2} \delta_{0}(C) \delta_{S_{1}\left(x_{0},\left(c_{1}, x_{1}\right), \ldots,\left(c_{k}, x_{k}\right)\right)}(A)+\frac{\alpha}{2} \delta_{1}(C) \delta_{S_{2}\left(x_{0},\left(c_{1}, x_{1}\right), \ldots,\left(c_{k}, x_{k}\right)\right)}(A) \\
& \quad+\beta \delta_{2}(C) \frac{\left|A \cap B_{\epsilon}\left(x_{k}\right)\right|}{\left|B_{\epsilon}\left(x_{k}\right)\right|}
\end{aligned}
$$

for any subset $C \subset \mathcal{C}$ and Borel subset $A \subset \Omega_{\epsilon}$ as long as $x_{k} \in \Omega$. If $x_{k} \notin \Omega$, the transition probability forces $x_{k+1}=x_{k}$.

The expected payoff is

$$
\mathbb{E}_{S_{1}, S_{2}}^{x_{0}}\left[F\left(x_{\tau}\right)\right]=\int_{H^{\infty}} F\left(x_{\tau}(\omega)\right) d \mathbb{P}_{S_{1}, S_{2}}^{x_{0}}
$$

when the game starts from $x_{0}$ and the players use strategies $S_{1}$ and $S_{2}$. The value of the game for Player 1 is given by

$$
u_{\epsilon}^{1}\left(x_{0}\right)=\sup _{S_{1}} \inf _{S_{2}} \mathbb{E}_{S_{1}, S_{2}}^{x_{0}}\left[F\left(x_{\tau}\right)\right]
$$

and the value of the game for Player 2 is given by

$$
u_{\epsilon}^{2}\left(x_{0}\right)=\inf _{S_{2}} \sup _{S_{1}} \mathbb{E}_{S_{1}, S_{2}}^{x_{0}}\left[F\left(x_{\tau}\right)\right],
$$

respectively. The game has a value that is, there exists a unique value function $u_{\epsilon}:=u_{\epsilon}^{1}=u_{\epsilon}^{2}$ (see [13] and [11]).

Since $\Omega$ is bounded, the game ends almost surely for any choice of strategies. This is true due to the fact that for $n_{0} \geq 1$ large enough, we have $n_{0} \epsilon>\operatorname{diam}(\Omega)$, and almost surely there will be infinitely many blocks of length $n_{0}$ consisting of solely random moves in the game.

Observe that the history $h_{k}$ contains all the information at the moment $k$, and since the strategies are a collection of Borel measurable functions from all possible histories, it is clear that the game process will not be a Markov process in general.

This version of the tug-of-war game has good symmetry properties, which we will utilize in the proofs. Other versions of tug-of-war games have been studied for example in [16] and [6] and a continuous time game in [2].

A rough outline of the connection between the version of the game considered in this paper and $p$-harmonic functions is the following. First, assume that we have a $p$-harmonic function in an open set $\Omega^{\prime} \supset \Omega$ with a nonvanishing gradient. Then, the $p$-harmonic function is real analytic, and Theorem 4.1 in [13] states that the game with probabilities (2.1) and with the values of the $p$ harmonic function on the boundary approximates the $p$-harmonic function in the game domain. The proof is based on the gradient strategy for the $p$-harmonic function and on the optional stopping theorem as well as on the asymptotic expansion in [12].

The general case requires game regularity of the boundary of the game domain. Then, it is possible to use a barrier argument to get estimates close to the boundary. By copying the strategies and utilizing the translation invariance of the game, the same estimates also holds in the 
interior of the game domain. Finally, a variant of the classical Arzelà-Ascoli's theorem provides a convergent subsequence. To prove that the limit is a viscosity solution to the homogeneous $p$-Laplace equation, a dynamic programming principle related to the game is applied (for more details about the principle, see, for example, [11]).

\section{Measure density condition implies game regularity}

We show in Theorem 3.7 that a uniform measure density condition implies game regularity for all $p>2$. To establish this, we first show in Lemma 3.3 a more attainable criterion for game regularity. Then in Theorem 3.6, we use a "cylinder walk" framework, introduced in [10], to obtain some important hitting probability estimates.

Definition 3.1. A point $y \in \partial \Omega$ satisfies a measure density condition if there is $c>0$ such that

$$
\left|\Omega^{c} \cap B_{r}(y)\right| \geq c\left|B_{r}(y)\right|
$$

for all $r>0$.

Definition 3.2. A point $y \in \partial \Omega$ is game regular, if for all $\delta>0$ and $\eta>0$, there exist $\delta_{0}>0$ and $\epsilon_{0}>0$ such that for all $\epsilon<\epsilon_{0}$ and $x_{0} \in B_{\delta_{0}}(y)$, there is a strategy $S_{1}^{*}$ for Player 1 such that

$$
\mathbb{P}_{S_{1}^{*}, S_{2}}^{x_{0}}\left(x_{\tau} \in B_{\delta}(y) \cap \Omega^{c}\right) \geq 1-\eta .
$$

If every boundary point of $\Omega$ is game regular, we say that $\Omega$ is game regular.

Roughly speaking, game regularity means that whenever the game starts near a boundary point $y$, Player 1 has a strategy to end the game near $y$ with a high probability. Next, we give a more attainable criterion to obtain game regularity. We modify the idea from [16], page 13 .

Lemma 3.3. A boundary point $y \in \partial \Omega$ is game regular if there exists a constant $\theta>0$ such that for all $\delta>0$, there are parameters $\epsilon_{0}>0$ and $\delta_{0}>0$ such that for all $\epsilon<\epsilon_{0}$ and $x_{0} \in B_{\delta_{0}}(y)$, there is a strategy $S_{1}^{*}$ for Player 1 such that

$$
\left.\mathbb{P}_{S_{1}^{*}, S_{2}}^{x_{0}} \text { (the game ends before exiting the ball } B_{\delta}(y)\right) \geq \theta .
$$

Proof. The idea of the proof is the following. By choosing $\delta_{0}>0$ small enough, we can start the game as near the point $y$ as we want, and in order to exit the ball $B_{\delta}(y)$, the game sequence has to exit all the concentric smaller balls inside $B_{\delta}(y)$ as well. The probability to exit all the concentric balls inside $B_{\delta}(y)$ can be estimated above via the uniform probability $\theta$; it is less than $(1-\theta)^{k}$, where $k$ is the amount of concentric balls inside $B_{\delta}(y)$. Thus, the probability to end the game near $y$ is close to one, when $k$ is big enough. 
To be more precise, let $\delta>0$ and $\eta>0$. Now, there are $\theta>0, \epsilon_{0,1}>0$ and $0<\delta_{0,1}<\delta$ such that for all $\epsilon<\epsilon_{0,1}$ and for all $x_{0} \in B_{\delta_{0,1}}(y)$, we have a strategy $S_{1}^{1}$ for Player 1 such that

$$
\left.\mathbb{P}_{S_{1}^{1}, S_{2}}^{x_{0}} \text { (the game ends before exiting the ball } B_{\delta}(y)\right) \geq \theta .
$$

We can assume that $\epsilon_{0,1}<\delta_{0,1} / 2$. Again similarly as above, for the constant $\delta_{0,1}-\epsilon_{0,1}$, there are $\epsilon_{0,2}>0$ and $0<\delta_{0,2}<\delta_{0,1} / 2$ such that for all $\epsilon<\epsilon_{0,2}$ and for all $x_{0} \in B_{\delta_{0,2}}(y)$, we have a strategy $S_{1}^{2}$ for Player 1 such that the probability to end the game before exiting the ball $B_{\delta_{0,1}-\epsilon_{0,1}}(y)$ is at least $\theta$. We can do this as many times we want. Let us do this $k \in \mathbb{N}$ times, where $k$ is such that

$$
(1-\theta)^{k} \leq \eta
$$

Define $\delta_{0}:=\delta_{0, k}$ and $\epsilon_{0}:=\min \left\{\epsilon_{0,1}, \ldots, \epsilon_{0, k}\right\}$, and fix any $x_{0} \in B_{\delta_{0}}(y)$ and $\epsilon<\epsilon_{0}$. We can assume that $\epsilon<\frac{1}{2} \min \left\{\delta_{0}, \delta_{0, k-1}-\delta_{0, k}, \ldots, \delta_{0,1}-\delta_{0,2}\right\}$ so that the game position cannot jump over many concentric balls during one turn. Denote the first time the game sequence exits $B_{\delta_{0, i-1}-\epsilon_{0, i-1}}(y)$ by $\tau^{i}:=\tau^{i}(\omega)$ for all $i \in\{1, \ldots, k\}$ with $\delta_{0,0}:=\delta$ and $\epsilon_{0,0}:=0$. Also, denote the set

$$
A_{i}:=\left\{\text { exits the ball } B_{\delta_{0, i-1}-\epsilon_{0, i-1}}(y) \text { before the game ends }\right\}
$$

for all $i \in\{1, \ldots, k\}$.

Recall that the game ends at the random time $\tau$. Define a strategy $S_{1}^{*}$ for Player 1 such that first, Player 1 uses the strategy $S_{1}^{k}$. If $\tau^{k}<\tau$, Player 1 starts to use the strategy $S_{1}^{k-1}$ after the stopping time $\tau^{k}$. Similarly, if $\tau^{k-1}<\tau$, Player 1 starts to use the strategy $S_{1}^{k-2}$ after the stopping time $\tau^{k-1}$. Thus, if it holds $0<\tau^{k}<\tau^{k-1}<\cdots<\tau^{1}<\tau$, after every stopping time $\tau^{i}$, Player 1 starts to use the strategy $S_{1}^{i-1}$ for all $i \in\{2, \ldots, k\}$ and for all game sequences $\omega \in H^{\infty}$. After the stopping time $\tau^{1}$, Player 1 does not change her strategy anymore. Observe that the earlier strategy $S_{1}^{i}$ does not affect the game after the first time the game sequence exits $B_{\delta_{0, i-1}-\epsilon_{0, i-1}}(y)$ for every $i \in\{2, \ldots, k\}$. Roughly this means that for every $i \in\{2, \ldots, k\}$, after the stopping time $\tau^{i}$, Player 1 forgets everything that has happened prior the time $\tau^{i}$.

Let $S_{2}$ be any strategy for Player 2 . The strategy $S_{2}$ can depend heavily on the past, so it could well be that our game process does not have any Markovian structure at any game round. However, the uniform $\theta$ is independent of the information available, so roughly, Player 2 cannot gain too much from the information of the past.

By the reasoning above, we can estimate iteratively

$$
\begin{aligned}
& \mathbb{P}_{S_{1}^{*}, S_{2}}^{x_{0}} \text { (exits the ball } B_{\delta}(y) \text { before the game ends) } \\
& \quad=\mathbb{E}_{S_{1}^{k}, S_{2}}^{x_{0}}\left[\chi_{A_{k}} \mathbb{E}_{S_{1}^{k-1}, S_{2}}^{x_{0}}\left[\prod_{l=1}^{k-1} \chi_{A_{l}} \mid \mathcal{F}_{\tau^{k}}\right]\right] \\
& \quad=\mathbb{E}_{S_{1}^{k}, S_{2}}^{x_{0}}\left[\chi_{A_{k}} \mathbb{E}_{S_{1}^{k-1}, S_{2}}^{x_{0}}\left[\chi_{A_{k-1}} \cdots \mathbb{E}_{S_{1}^{1}, S_{2}}^{x_{0}}\left[\chi_{A_{1}} \mid \mathcal{F}_{\tau^{2}}\right] \cdots \mid \mathcal{F}_{\tau^{k}}\right]\right] \\
& \leq(1-\theta)^{k} \leq \eta .
\end{aligned}
$$


This implies that

$$
\begin{aligned}
\mathbb{P}_{S_{1}^{*}, S_{2}}^{x_{0}}\left(x_{\tau} \in B_{\delta}(y) \cap \Omega^{c}\right) & \geq \mathbb{P}_{S_{1}^{*}, S_{2}}^{x_{0}}\left(\text { the game ends before exiting } B_{\delta}(y)\right) \\
& \geq 1-\eta
\end{aligned}
$$

Thus, we have shown the game regularity.

To see that the uniform measure density condition implies game regularity, we need a "cylinder walk" framework.

Cylinder walk. Set the constants $\alpha, \beta>0$ with $\alpha+\beta=1$ as before in (2.1), and fix the cylinder size $r>0$. Consider the following random walk (called the "cylinder walk") in a $(n+1)$ dimensional cylinder $B_{r}(0) \times[0, r]$. Suppose that we are at a point $\left(x_{j}, t_{j}\right) \in B_{r}(0) \times[0, r]$. Next, we move to the point $\left(x_{j}, t_{j}-\epsilon\right)$ with the probability $\alpha / 2$ and to the point $\left(x_{j}, t_{j}+\epsilon\right)$ with the probability $\alpha / 2$. With the probability $\beta$ we move to the point $\left(x_{j+1}, t_{j}\right)$, where $x_{j+1}$ is chosen from the ball $B_{\epsilon}\left(x_{j}\right)$ according to the uniform distribution.

We have the following estimate for the probability that the cylinder walk exits the cylinder through its bottom; the proof is in the Appendix of the paper [10].

Lemma 3.4. Let us start the cylinder walk from the point $(0, t)$ with $0<t<r$. Then, the probability that the walk exits the cylinder through its bottom is at least

$$
1-C_{n, p}(t+\epsilon) / r
$$

for all $\epsilon>0$ small enough.

Assume that the origin $0 \in \mathbb{R}^{n+1}$ at the bottom of the cylinder belongs to the set $\partial \Omega \times\{0\}$ and that this boundary point satisfies the measure density condition. The set $\Omega \cap B_{r}(0) \times\{0\} \subset$ $B_{r}(0) \times[0, r]$. We are interested in the probability that the cylinder walk exits through the bottom and in addition, at the first time the walk hits the bottom, the process is in the complement of the set $\Omega$. Since the origin satisfies the measure density condition, the complement has some positive Lebesgue measure. This suggests that the event we are interested in could have some positive probability measure.

The cylinder walk can be constructed by combining three independent random constructions. There is a "horizontal" random walk with the initial position $\tilde{x}_{0}=x \in B_{r}(0)$. The point $\tilde{x}_{j+1}$ is chosen according to the uniform distribution in the ball $B_{\epsilon}\left(\tilde{x}_{j}\right) \subset \mathbb{R}^{n}$ for all $j \geq 0$. Further, there is a "vertical" random walk in the real axis with steps $+\epsilon$ or $-\epsilon$ and with the initial position $\left.\tilde{t}_{0}=t \in\right] 0, r\left[\right.$. For all $j \geq 0$, the next positions are $\tilde{t}_{j+1}=\tilde{t}_{j}+\epsilon$ or $\tilde{t}_{j+1}=\tilde{t}_{j}-\epsilon$ both with probability $\frac{1}{2}$. In addition, there is the increasing sequence

$$
U_{j}=\sum_{m=1}^{j} \operatorname{Ber}_{m},
$$


where the $\operatorname{Ber}_{m}$ 's are independent Bernoulli variables with $\operatorname{Ber}_{m}(\omega) \in\{0,1\}$ and $\mathbb{P}\left(\operatorname{Ber}_{m}=1\right)=$ $\alpha$. Therefore, a copy of the cylinder walk is obtained by letting for $j \geq 0$

$$
t_{j}=\tilde{t}_{U_{j}}, \quad x_{j}=\tilde{x}_{j-U_{j}}
$$

Let $\tau_{g}$ stand for the first moment $t_{j}$ exits the cylinder through its bottom or top, that is, the first $j$ such that $\left.t_{j} \in \mathbb{R} \backslash\right] 0, r$. Also, let $\tilde{\tau}_{g}$ stand for the first moment $\tilde{t}_{j}$ exits the cylinder through its bottom or top. Here, the subindex $g$ refers to a "good exit".

We assume that $x=0$. First, let us study the properties of the function $\tau_{g}-U_{\tau_{g}}=\tau_{g}-\tilde{\tau}_{g}$. The random variable $\tau_{g}-\tilde{\tau}_{g}$ is the number of times a random horizontal movement has occurred at the first moment the cylinder walk hits the bottom or top. The proof of the lemma below is in the Appendix for completeness.

Lemma 3.5. Let $\tau_{g}, \tilde{\tau}_{g}, \alpha, \beta$ and $r$ be as above, and let $n_{0} \geq 1$ and $\left.\gamma \in\right] 0,1[$. Then, there is a universal constant $C:=C_{n_{0}, n, p, \gamma}>1$ such that for all $a>0, C \epsilon \leq t<r / 2$ and $\epsilon$ small enough it holds

$$
\begin{aligned}
\mathbb{P}\left(\tau_{g}-\tilde{\tau}_{g} \geq n_{0}\right) & \geq 1-\gamma \quad \text { and } \\
\mathbb{P}\left(\tau_{g}-\tilde{\tau}_{g} \geq a \epsilon^{-2}\right) & \leq 1-\frac{2}{\sqrt{2 \pi}} \int_{\frac{t}{\sqrt{a}} v_{n, p}}^{\infty} e^{-\frac{s^{2}}{2}} d s+\gamma+\mathcal{O}(\epsilon)
\end{aligned}
$$

with the constant

$$
v_{n, p}:=2 \sqrt{\frac{\beta+0.01 \alpha}{0.99 \alpha}} .
$$

For any $a>0, n_{0} \geq 1$ and $\left.\gamma \in\right] 0,1[$ the inequalities (3.1) and (3.2) yield

$$
\begin{aligned}
\mathbb{P}\left(n_{0} \leq \tau_{g}-\tilde{\tau}_{g}<a \epsilon^{-2}\right) & \geq \mathbb{P}\left(\tau_{g}-\tilde{\tau}_{g} \geq n_{0}\right)-\mathbb{P}\left(\tau_{g}-\tilde{\tau}_{g} \geq a \epsilon^{-2}\right) \\
& \geq \frac{2}{\sqrt{2 \pi}} \int_{\frac{t}{\sqrt{a}} v_{n, p}}^{\infty} e^{-\frac{s^{2}}{2}} d s-2 \gamma-\mathcal{O}(\epsilon)
\end{aligned}
$$

for all $C \epsilon \leq t<r / 2$ and $\epsilon$ small enough with a large $C>1$ independent of $\epsilon$. Observe that

$$
\frac{2}{\sqrt{2 \pi}} \int_{\frac{t}{\sqrt{a}} v_{n, p}}^{\infty} e^{-\frac{s^{2}}{2}} d s \rightarrow 1
$$

as $t \rightarrow 0$. Thus, the inequality (3.3) points out that for the cylinder walk started from the height $C \epsilon \leq t<r_{0}$, the random variable $\tau_{g}-\tilde{\tau}_{g}$ is very likely between the times $n_{0}$ and $a \epsilon^{-2}$ for all $r_{0}$, $\gamma$ and $\epsilon$ small enough and fixed $n_{0} \geq 1$ and $a>0$.

Next, we concentrate on the distribution of the random variable $\tilde{x}_{k}$. Assume that $Z$ is a random vector with the uniform distribution in the ball $B_{\epsilon}(0) \subset \mathbb{R}^{n}$. The density of the random vector $Z$ is

$$
f_{Z}(x)=\frac{1}{\left|B_{\epsilon}(0)\right|} \chi_{B_{\epsilon}(0)}(x) .
$$


We denote the measure of the unit ball by $\omega_{n}:=\left|B_{1}(0)\right|$. Let $k_{0}:=k_{0, n}>2$ denote the constant in Lemma A.4 and fix any $k \geq k_{0}$. For the density of the random variable $\tilde{x}_{k}=\sum_{i=1}^{k} Z_{i}$, where the random vectors $Z_{i}$ are independent and distributed as $Z$, we use the notation $f_{k}:=f_{\sum_{i=1}^{k} Z_{i}}$. The density $f_{k}$ is a decreasing radial function. In the Appendix, we have derived in (A.8) and (A.10) the following estimates: There are constants $C_{n}>0$ and $C_{1}>0$ such that

$$
f_{k}(0) \leq C_{n}\left(\frac{1}{\sqrt{k} \epsilon}\right)^{n}
$$

and

$$
f_{k}\left(C_{*} \sqrt{k} \epsilon\right) \geq\left(\frac{1}{C_{1}}\right)^{n}\left(\frac{0.99}{\omega_{n}}-C_{n}\left(C_{*}\right)^{n}\right)\left(\frac{1}{\sqrt{k} \epsilon}\right)^{n}
$$

for all $\left.C_{*} \in\right] 0, C_{1}[$. By the comment after the statement of Lemma A.4 in the Appendix, we have

$$
f_{k}\left(C_{*} \sqrt{k} \epsilon\right) \geq \zeta\left(\frac{1}{\sqrt{k} \epsilon}\right)^{n}
$$

for some $\zeta:=\zeta_{n}>0$, if we choose $C_{*}>0$ so small that

$$
C_{*}<\left(\frac{0.99}{\omega_{n} C_{n}}\right)^{1 / n} .
$$

Let $\tau_{b}$ stand for the first $j$ when $\left|x_{j}\right|$ reaches $[r, \infty[$. Here, the subindex $b$ refers to a "bad exit". Recall that the origin at the bottom of the cylinder satisfies the measure density condition. Let $C_{n, p}>0$ denote the constant in Lemma 3.4, and for all $\delta>0$, denote

$$
A_{\delta}:=B_{\delta}(0) \cap \Omega^{c} .
$$

Theorem 3.6. Consider the cylinder $B_{\delta / 3}(0) \times[0, \delta / 3]$ for any fixed $\delta>0$. Then, there exist constants $\theta:=\theta_{n, p}>0, \epsilon_{0}:=\epsilon_{0, n, p, \delta}>0$ and $\delta_{0}:=\delta_{0, n, p, \delta}>0$ such that

$$
\mathbb{P}\left(\tau_{b} \leq \tau_{g} \text { or } t_{\tau_{g}} \geq \delta / 3 \text { or } x_{\tau_{g}} \notin A_{\delta / 3}\right) \leq 1-\theta
$$

for all $\epsilon<\epsilon_{0}$ whenever the cylinder walk starts from the point $(0, t)$ for some $0<t \leq \delta_{0}$.

Proof. To establish the result, we use the inequality (3.3) to estimate how many times it is likely that a random horizontal movement has occurred at the first time the cylinder walk hits the bottom. Then, we use the estimate (3.4) and the fact that vertical and horizontal movements are independent to estimate the probability that we are in the complement of the set $\Omega$ at the first time the walk exits the cylinder through its bottom.

Let $0<\lambda<1$, where the exact value of $\lambda$ will be fixed later. Define

$$
\delta_{0}:=\frac{\delta \lambda}{3 C_{n, p}}
$$


and start the cylinder walk from the point $(0, t)$ for some $0<t \leq \delta_{0}$ in the cylinder $B_{\delta / 3}(0) \times$ $[0, \delta / 3]$.

We recall the constant $k_{0}:=k_{0, n}>2$ from Lemma A.4. In addition, let $C:=C_{n_{0}, n, p, \gamma}>1$ be the constant from Lemma 3.5 with $n_{0}=k_{0}$ and $0<\gamma<1$ defined later. First, assume that $t<C \epsilon$. Then, the number of $\epsilon$-steps required to reach the bottom from $t$ is less than the universal constant $C$. Hence, the probability that the cylinder walk exits the cylinder through its bottom and in addition, it holds $x_{\tau_{g}}=0$, is greater than or equal to $(\alpha / 2)^{C}$. From this the statement immediately follows in the case $t<C \epsilon$.

Next, assume that $t \geq C \epsilon$. Lemma 3.4 states that

$$
\mathbb{P}\left(\tau_{b} \leq \tau_{g} \text { or } t_{g} \geq \delta / 3\right) \leq 3 C_{n, p} \delta^{-1}(t+\epsilon) \leq 3 C_{n, p} \delta^{-1}\left(\delta_{0}+\epsilon\right) .
$$

Therefore, we have by (3.6) that

$$
\mathbb{P}\left(\tau_{b} \leq \tau_{g} \text { or } t_{\tau_{g}} \geq \delta / 3 \text { or } x_{\tau_{g}} \notin A_{\delta / 3}\right) \leq \mathcal{O}(\epsilon)+\lambda+1-\mathbb{P}\left(x_{\tau_{g}} \in A_{\delta / 3}\right) .
$$

The inequality (3.3) and the remark after suggest the estimate

$$
\begin{aligned}
\mathbb{P}\left(x_{\tau_{g}} \in A_{\delta / 3}\right) & =\mathbb{P}\left(\tilde{x}_{\tau_{g}-\tilde{\tau}_{g}} \in A_{\delta / 3}\right) \\
\geq & \mathbb{P}\left(\tilde{x}_{\tau_{g}-\tilde{\tau}_{g}} \in A_{\delta / 3} \text { and } k_{0} \leq \tau_{g}-\tilde{\tau}_{g}<\delta^{2} \epsilon^{-2}\right) \\
& =\sum_{k=k_{0}}^{\left\lfloor\delta^{2} \epsilon^{-2}\right\rfloor} \mathbb{P}\left(\tilde{x}_{\tau_{g}-\tilde{\tau}_{g}} \in A_{\delta / 3} \text { and } \tau_{g}-\tilde{\tau}_{g}=k\right) .
\end{aligned}
$$

Denote the index set

$$
I:=\left\{k_{0}, k_{0}+1, \ldots,\left\lfloor\delta^{2} \epsilon^{-2}\right\rfloor\right\} .
$$

Since the random variables $\tilde{x}_{k}$ and $\tau_{g}-\tilde{\tau}_{g}$ are independent for all $k \in I$, we have

$$
\sum_{k \in I} \mathbb{P}\left(\tilde{x}_{\tau_{g}-\tilde{\tau}_{g}} \in A_{\delta / 3} \text { and } \tau_{g}-\tilde{\tau}_{g}=k\right)=\sum_{k \in I} \mathbb{P}\left(\tilde{x}_{k} \in A_{\delta / 3}\right) \mathbb{P}\left(\tau_{g}-\tilde{\tau}_{g}=k\right) .
$$

Let $k \in I$ and choose the constant $C_{*}>0$ as in (3.5). We may assume that $C_{*}<1 / 3$. Because $\sqrt{k} \epsilon<\delta$ and the density $f_{k}$ is a decreasing radial function, we can calculate

$$
\mathbb{P}\left(\tilde{x}_{k} \in A_{\delta / 3}\right) \geq \mathbb{P}\left(\tilde{x}_{k} \in B_{C_{*} \sqrt{k} \epsilon}(0) \cap \Omega^{c}\right) \geq f_{k}\left(C_{*} \sqrt{k} \epsilon\right)\left|B_{C_{*} \sqrt{k} \epsilon}(0) \cap \Omega^{c}\right| .
$$

By using the estimate (3.4) and the uniform measure density condition, we obtain

$$
\begin{aligned}
& f_{k}\left(C_{*} \sqrt{k} \epsilon\right)\left|B_{C_{*} \sqrt{k} \epsilon}(0) \cap \Omega^{c}\right| \\
& \geq\left(\frac{1}{C_{1}}\right)^{n}\left(\frac{0.99}{\omega_{n}}-C_{n}\left(C_{*}\right)^{n}\right)\left(\frac{1}{\sqrt{k} \epsilon}\right)^{n} c\left|B_{C_{*} \sqrt{k} \epsilon}(0)\right| \\
& =\omega_{n} c\left(\frac{C_{*}}{C_{1}}\right)^{n}\left(\frac{0.99}{\omega_{n}}-C_{n}\left(C_{*}\right)^{n}\right)=: \hat{C}_{n},
\end{aligned}
$$


where the constant $c>0$ comes from the uniform measure density condition. This together with the inequality (3.3) yield

$$
\begin{aligned}
& \sum_{k \in I} \mathbb{P}\left(\tilde{x}_{k} \in A_{\delta / 3}\right) \mathbb{P}\left(\tau_{g}-\tilde{\tau}_{g}=k\right) \\
& \quad \geq \omega_{n} c\left(\frac{C_{*}}{C_{1}}\right)^{n}\left(\frac{0.99}{\omega_{n}}-C_{n}\left(C_{*}\right)^{n}\right) \mathbb{P}\left(k_{0} \leq \tau_{g}-\tilde{\tau}_{g}<\delta^{2} \epsilon^{-2}\right) \\
& \quad \geq \hat{C}_{n} \frac{2}{\sqrt{2 \pi}} \int_{\frac{\lambda}{3} \tilde{C}_{n, p}}^{\infty} e^{-\frac{s^{2}}{2}} d s-2 \gamma \hat{C}_{n}-\mathcal{O}(\epsilon)
\end{aligned}
$$

with the constant

$$
\tilde{C}_{n, p}:=\frac{2}{C_{n, p}} \sqrt{\frac{\beta+0.01 \alpha}{0.99 \alpha}} .
$$

Therefore, we have shown

$$
\begin{aligned}
& \mathbb{P}\left(\tau_{b} \leq \tau_{g} \text { or } t_{\tau_{g}} \geq \delta / 3 \text { or } x_{\tau_{g}} \notin A_{\delta / 3}\right) \\
& \quad \leq 1-\hat{C}_{n} \frac{2}{\sqrt{2 \pi}} \int_{\frac{1}{3} \tilde{C}_{n, p}}^{\infty} e^{-\frac{s^{2}}{2}} d s+\lambda+2 \gamma \hat{C}_{n}+\mathcal{O}(\epsilon) \\
& \quad \leq 1-\frac{1}{2} \hat{C}_{n} \frac{2}{\sqrt{2 \pi}} \int_{\frac{1}{3} \tilde{C}_{n, p}}^{\infty} e^{-\frac{s^{2}}{2}} d s
\end{aligned}
$$

for all $C \epsilon \leq t \leq \delta_{0}$ and $\epsilon, \lambda$ and $\gamma$ small enough. Thus, this concludes the case $t \geq C \epsilon$. To combine the cases, we define

$$
\theta:=\min \left\{\frac{1}{2} \hat{C}_{n} \frac{2}{\sqrt{2 \pi}} \int_{\frac{1}{3} \tilde{C}_{n, p}}^{\infty} e^{-\frac{s^{2}}{2}} d s,(\alpha / 2)^{C}\right\} .
$$

Consequently, the proof is complete.

If Player 1 plays by canceling the moves of the other player, we obtain Theorem 3.7. Observe that this strategy is not optimal for Player 1 in the sense that Player 1 also tries to cancel the moves that might benefit her.

The cancellation strategy was introduced in the paper [10] to prove Harnack's inequality for $p$-harmonic functions via tug-of-war games. In addition, the cancellation strategy can be used to prove regularity properties for viscosity solutions of the inhomogeneous $p$-Laplace equation (see [18]).

Theorem 3.7. If $y \in \partial \Omega$ satisfies the measure density condition, then it is game regular for $p>2$.

Proof. To establish the result, our aim is to use Lemma 3.3 and therefore, to find a uniform lower bound for the probability that the game ends before exiting a given ball. If Player 1 plays 
by canceling the moves of the other player, the lower bound $\theta>0$ for the probability is obtained by using Theorem 3.6.

We can clearly assume that $y=0$. Let $\delta>0$, and consider the cylinder $B_{\delta / 3}(0) \times[0, \delta / 3]$. Define a constant $\delta_{0}$ as in (3.6), and find $\epsilon_{0}>0$ and $\lambda>0$ small enough such that we can apply Theorem 3.6. Let $x_{0} \in B_{\delta_{0}}(0)$ and $\epsilon<\epsilon_{0}$. At every moment, we can divide the game position as a sum of vectors

$$
x_{0}+\sum_{k \in I_{1}} v_{k}^{1}+\sum_{k \in I_{2}} v_{k}^{2}+\sum_{k \in I_{3}} v_{k}^{3}
$$

Here, $I_{1}$ denotes the indices of rounds when Player 1 has moved with the vectors $v_{k}^{1}$ as her moves. Similarly, Player 2 has moved in the indices of rounds $I_{2}$ with the moves $v_{k}^{2}$ as his moves. The random movements have occurred in the indices of rounds $I_{3}$, and these random movements are denoted by $v_{k}^{3}$.

Let

$$
M:=2\left\lceil\frac{\left|x_{0}\right|}{\epsilon}\right\rceil,
$$

where the factor 2 is due to the fact that the players cannot step to the boundary of $B_{\epsilon}\left(x_{j}\right)$ for any $j$. Define the following strategy $S_{1}^{*}$ for Player 1 for the game that starts from $x_{0}$. She always tries to cancel the earliest move of Player 2 which she has not yet been able to cancel. If all the moves at that moment are cancelled and she wins the coin toss, she moves the game point by the vector

$$
-\epsilon / 2 \frac{x_{0}}{\left|x_{0}\right|} .
$$

She does this until she has won $M-1$ more coin tosses than Player 2. If she wins her $M$ th more coin toss, her move will be such that the game position is

$$
\sum_{k \in I_{3}} v_{k}^{3}
$$

after the move. Observe that the game, with the strategy $S_{1}^{*}$, is related to the cylinder walk, when we start the cylinder walk from the point $(0, M \epsilon / 2)$ with $M \epsilon / 2 \rightarrow\left|x_{0}\right|<\delta_{0}$ as $\epsilon \rightarrow 0$.

Let us define three conditions for the game sequences of the game:

(A) Player 1 has won the coin toss $M$ more times than Player 2, and at the moment this happens, the game sequence is in the set $\Omega^{c}$.

(B) Player 2 has won the coin toss at least $\frac{\delta}{3 \epsilon}$ more times than Player 1.

(C) $\left|\sum_{k \in I_{3}} v_{k}^{3}\right| \geq \frac{\delta}{3}$.

We are interested in the following event

$$
\mathbf{X}:=\{\text { the condition (A) happens before conditions }(\mathbf{B}) \text { and }(\mathbf{C})\},
$$

and Theorem 3.6 states that there is a constant $\theta:=\theta_{n, p}>0$ such that

$$
\mathbb{P}_{S_{1}^{*}, S_{2}}(\mathbf{X}) \geq \theta .
$$


Now, we can estimate

$$
\mathbb{P}_{S_{1}^{*}, S_{2}}\left(\text { the game ends before exiting the ball } B_{\delta}(0)\right) \geq \mathbb{P}_{S_{1}^{*}, S_{2}}(\mathbf{X}) \text {. }
$$

Above, we also used the fact that the game sequences for which the game has ended before Player 1 has won $M$ more coin tosses than Player 2 are good for our purposes. To finish the proof, we can use Lemma 3.3, and thus the proof is complete.

It is worth mentioning that in the case $p>n$, every point becomes game regular. This is proved in [16], and the same also holds for the version of the game considered in this paper. Roughly, as $p$ increases, the probability for the player to end the game before exiting a given ball increases.

\section{Appendix: Hitting probabilities for a cylinder walk}

Fix the cylinder size $r>0$. The cylinder walk in a cylinder $B_{r}(0) \times[0, r] \subset \mathbb{R}^{n+1}$ can be constructed by combining three independent random constructions. There is a "horizontal" random walk with the initial position $\tilde{x}_{0}=x \in B_{r}(0)$. The point $\tilde{x}_{j+1}$ is chosen according to the uniform distribution in the ball $B_{\epsilon}\left(\tilde{x}_{j}\right) \subset \mathbb{R}^{n}$ for all $j \geq 0$. Further, there is a "vertical" random walk in the real axis with steps $+\epsilon$ or $-\epsilon$ and with the initial position $\left.\tilde{t}_{0}=t \in\right] 0, r[$. The next positions are $\tilde{t}_{j+1}=\tilde{t}_{j}+\epsilon$ or $\tilde{t}_{j+1}=\tilde{t}_{j}-\epsilon$ both with probability $\frac{1}{2}$ for all $j \geq 0$. In addition, there is the increasing sequence

$$
U_{j}=\sum_{m=1}^{j} \operatorname{Ber}_{m}
$$

where the $\operatorname{Ber}_{m}$ 's are independent Bernoulli variables with $\operatorname{Ber}_{m}(\omega) \in\{0,1\}$ and $\mathbb{P}\left(\operatorname{Ber}_{m}=1\right)=$ $\alpha \in] 0,1[$. Thus, a copy of the cylinder walk is obtained by letting for $j \geq 0$

$$
t_{j}=\tilde{t}_{U_{j}}, \quad x_{j}=\tilde{x}_{j-U_{j}}
$$

Let $\tau_{g}$ stand for the first moment $t_{j}$ exits the cylinder through its bottom or top, and let $\tilde{\tau}_{g}$ stand for the first moment $\tilde{t}_{j}$ exits the cylinder through its bottom or top.

Recall Hoeffding's (or Azuma's or Bernstein's) inequality for a sum of independent and identically distributed random variables (see, for example, [7], page 198).

Theorem A.1. Let $Y_{m}$ be independent and identically distributed symmetric $\mathbb{R}^{n}$-valued random variables, $m \in\{1,2, \ldots, N\}$, that are uniformly bounded: $\left|Y_{m}\right| \leq b$ almost surely for all $m$. Then,

$$
\mathbb{P}\left(\max _{1 \leq m \leq N}\left|\sum_{i=1}^{m} Y_{i}\right| \geq \lambda\right) \leq 4 n \exp \left(-\frac{\lambda^{2}}{2 N b^{2} n}\right) .
$$


In the theorem above, the factor 4 instead of 2 comes from the use of Levy-Kolmogorov's inequality (see, for example, [19], page 397)

$$
\mathbb{P}\left(\max _{1 \leq m \leq N}\left|\sum_{i=1}^{m} Y_{i}\right| \geq \lambda\right) \leq 2 \mathbb{P}\left(\left|\sum_{i=1}^{N} Y_{i}\right| \geq \lambda\right) .
$$

We assume that $x=0$, and denote $\beta=1-\alpha$.

Lemma A.2. Let $\tau_{g}$ and $\tilde{\tau}_{g}$ be as above, $n_{0} \geq 1$ and $\left.\gamma \in\right] 0,1[$. Then, there is a constant $C:=$ $C_{n_{0}, n, p, \gamma}>0$ such that it holds

$$
\begin{aligned}
\mathbb{P}\left(\tilde{\tau}_{g} \geq n_{0}\right) & \geq 1-\gamma \quad \text { and } \\
\mathbb{P}\left(\tau_{g}-\tilde{\tau}_{g} \geq n_{0}\right) & \geq 1-\gamma
\end{aligned}
$$

for all $C \epsilon \leq t<r / 2$ and $\epsilon<r /(4 C)$.

Proof. The vertical movement consists of the moves $+\epsilon$ or $-\epsilon$ in the real axis. Let $Y_{i}$ be independent and identically distributed random variables with $Y_{i}(\omega) \in\{-\epsilon, \epsilon\}$ and $\mathbb{P}\left(Y_{i}=\epsilon\right)=$ $\mathbb{P}\left(Y_{i}=-\epsilon\right)=\frac{1}{2}$ for all $i$. Assume $t<r / 2$, and recall the cylinder size $B_{r}(0) \times[0, r]$. Now, it holds

$$
\begin{aligned}
\mathbb{P}\left(\tilde{\tau}_{g} \geq n_{0}\right) & =\mathbb{P}\left(\max _{k<n_{0}} \sum_{i=1}^{k} Y_{i}<\min \{t, r-t\}\right) \\
& =1-\mathbb{P}\left(\max _{k<n_{0}} \sum_{i=1}^{k} Y_{i} \geq t\right) .
\end{aligned}
$$

Random variables $Y_{i}$ are bounded, $\left|Y_{i}\right| \leq \epsilon$ for all $i \geq 1$. By using Hoeffding's inequality that is, Theorem A.1, we can deduce that for $C>0$ and $t \geq C \epsilon$ it holds

$$
\mathbb{P}\left(\max _{k<n_{0}} \sum_{i=1}^{k} Y_{i} \geq t\right) \leq 4 \exp \left(-\frac{t^{2}}{2 n_{0} \epsilon^{2}}\right) \leq 4 \exp \left(-\frac{C^{2}}{2 n_{0}}\right) .
$$

Consequently, there is $\bar{C}:=\bar{C}_{n_{0}, \gamma}>1$ large enough such that (A.1) holds for all $\bar{C} \epsilon \leq t<r / 2$ and $\epsilon<r /(4 \bar{C})$.

For the second part, let us consider the event

$$
B:=\left\{0.99 \alpha \tau_{g}<\tilde{\tau}_{g}<(\alpha+\beta / 2) \tau_{g}\right\} .
$$

For any $j_{0} \geq 1$, denote the sets

$$
\begin{aligned}
& B^{*}:=\left\{U_{j}<(\alpha+\beta / 2) j \text { for all } j \geq j_{0}\right\} \text { and } \\
& B_{*}:=\left\{U_{j}>0.99 \alpha j \text { for all } j \geq j_{0}\right\} .
\end{aligned}
$$


Again, apply Hoeffding's inequality with $Y_{m}=\operatorname{Ber}_{m}-\alpha, \lambda=j \beta / 2, b=1$ and $N=j$ to get

$$
\begin{aligned}
\mathbb{P}\left(U_{j} \geq(\alpha+\beta / 2) j\right) & =\mathbb{P}\left(U_{j}-\alpha j \geq j \beta / 2\right) \leq \mathbb{P}\left(\left|U_{j}-\alpha j\right| \geq j \beta / 2\right) \\
& \leq 4 \exp \left(-\frac{1}{8} \beta^{2} j\right) .
\end{aligned}
$$

In a similar fashion, we can calculate

$$
\mathbb{P}\left(U_{j} \leq 0.99 \alpha j\right) \leq 4 \exp \left(-\frac{\alpha^{2} j}{2 \cdot 10^{4}}\right) .
$$

Thus by choosing $j_{0}$ large enough and summing over all indices, we get

$$
\begin{aligned}
\mathbb{P}\left(\left(B^{*}\right)^{c}\right) & =\mathbb{P}\left(U_{j} \geq(\alpha+\beta / 2) j \text { for some } j \geq j_{0}\right) \\
& \leq \sum_{j \geq j_{0}} \mathbb{P}\left(U_{j} \geq(\alpha+\beta / 2) j\right) \\
& \leq \sum_{j \geq j_{0}} 4 \exp \left(-\frac{1}{8} \beta^{2} j\right) \leq \frac{\gamma}{8} .
\end{aligned}
$$

By a similar argument, it holds

$$
\mathbb{P}\left(B_{*}\right) \geq 1-\gamma / 8
$$

for $j_{0}$ large enough. Hence, we choose a large index $j_{0}:=j_{0, n, p, \gamma}$ such that

$$
\mathbb{P}\left(B_{*} \text { and } B^{*}\right) \geq \mathbb{P}\left(B_{*}\right)-\mathbb{P}\left(\left(B^{*}\right)^{c}\right) \geq 1-\gamma / 4 .
$$

Observe that

$$
\left\{B_{*} \text { and } B^{*} \text { and } \tau_{g} \geq j_{0}\right\} \subset B \text {. }
$$

Therefore, we get

$$
\mathbb{P}(B) \geq \mathbb{P}\left(\tau_{g} \geq j_{0}\right)-\gamma / 4
$$

Since $\tau_{g} \geq \tilde{\tau}_{g}$ always, we have $\left\{\tilde{\tau}_{g} \geq j_{0}\right\} \subset\left\{\tau_{g} \geq j_{0}\right\}$. Combining this with a similar argument to (A.1), we can deduce that there is $\tilde{C}:=\tilde{C}_{j_{0}, \gamma}>1$ large enough such that for all $\tilde{C} \epsilon \leq t<r / 2$ and $\epsilon<r /(4 \tilde{C})$ it holds

$$
\mathbb{P}(B) \geq 1-\gamma / 2 .
$$

By a direct calculation, we have

$$
B \subset\left\{\frac{\beta}{2 \alpha+\beta} \tilde{\tau}_{g}<\tau_{g}-\tilde{\tau}_{g}<\frac{\beta+0.01 \alpha}{0.99 \alpha} \tilde{\tau}_{g}\right\} .
$$


Therefore, we obtain by using (A.4)

$$
\begin{aligned}
\mathbb{P}\left(\tau_{g}-\tilde{\tau}_{g} \geq n_{0}\right) & \geq \mathbb{P}\left(\frac{\beta}{2 \alpha+\beta} \tilde{\tau}_{g} \geq n_{0} \text { and } B\right) \\
& =\mathbb{P}\left(\tilde{\tau}_{g} \geq(2 \alpha+\beta) n_{0}(\beta)^{-1} \text { and } B\right) \\
& \geq \mathbb{P}\left(\tilde{\tau}_{g} \geq(2 \alpha+\beta) n_{0}(\beta)^{-1}\right)-\gamma / 2
\end{aligned}
$$

for all $\tilde{C} \epsilon \leq t<r / 2$ and $\epsilon<r /(4 \tilde{C})$. Thus, this estimate and a similar argument to (A.1) imply that there is $C:=C_{n_{0}, n, p, \gamma}>\max \{\bar{C}, \tilde{C}\}>1$ large enough such that (A.2) holds for all $C \epsilon \leq$ $t<r / 2$ and $\epsilon<r /(4 C)$.

Lemma A.3. Let $\tau_{g}, \tilde{\tau}_{g}, r$ and $\alpha, \beta>0$ such that $\alpha+\beta=1$ be as at the beginning of the Appendix. In addition, let $C:=C_{n_{0}, n, p, \gamma}>1$ be the constant from Lemma A.2 for $\left.\gamma \in\right] 0,1[$ and $n_{0} \geq 1$. Then for all $a>0, C \epsilon \leq t<r / 2$ and $\epsilon$ small enough, we have

$$
\mathbb{P}\left(\tau_{g}-\tilde{\tau}_{g} \geq a \epsilon^{-2}\right) \leq 1-\frac{2}{\sqrt{2 \pi}} \int_{\frac{t}{\sqrt{a}} v_{n, p}}^{\infty} e^{-\frac{s^{2}}{2}} d s+\gamma+\mathcal{O}(\epsilon)
$$

with the constant

$$
v_{n, p}:=2 \sqrt{\frac{\beta+0.01 \alpha}{0.99 \alpha}} .
$$

Proof. By using the inequality (A.4) and the inclusion after it, we can deduce

$$
\begin{aligned}
\mathbb{P}\left(\tau_{g}-\tilde{\tau}_{g} \geq a \epsilon^{-2}\right) & \leq \mathbb{P}\left(\tau_{g}-\tilde{\tau}_{g} \geq a \epsilon^{-2} \text { and } B\right)+\mathbb{P}\left(B^{c}\right) \\
& \leq \mathbb{P}\left(\tilde{\tau}_{g} \geq \frac{0.99 \alpha a}{(\beta+0.01 \alpha) \epsilon^{2}}\right)+\gamma
\end{aligned}
$$

for all $C \epsilon \leq t<r / 2$ and $\epsilon<r /(4 C)$ with the set $B$ defined in (A.3).

We estimate the probability of the event $\left\{\tilde{\tau}_{g} \geq d \epsilon^{-2}\right\}$ for all $d>0$. Consider the following independent and identically distributed random variables: $Z_{i}(\omega) \in\{1,-1\}, \mathbb{P}\left(Z_{i}=-1\right)=\mathbb{P}\left(Z_{i}=\right.$ $1)=\frac{1}{2}$ and $\mathbb{E}\left[Z_{i}\right]^{2}=1$ for all $i \geq 1$. For these random variables, we have the following equality (see, for example, [7], page 351)

$$
\mathbb{P}\left(\max _{1 \leq m \leq N} \sum_{i=1}^{m} Z_{i} \geq l\right)=2 \mathbb{P}\left(\sum_{i=1}^{N} Z_{i} \geq l\right)-\mathbb{P}\left(\sum_{i=1}^{N} Z_{i}=l\right)
$$

for all integers $N \geq 1$ and $l \geq 1$. Further, since $\mathbb{E}\left|Z_{i}\right|^{3}=1<\infty$ for all $i \geq 1$, we can use the Berry-Esseen theorem to determine the speed in the central limit theorem (see, for example, [19], page 63), and thus

$$
2 \mathbb{P}\left(\sum_{i=1}^{N} Z_{i} \geq l \sqrt{N}\right)-\mathbb{P}\left(\sum_{i=1}^{N} Z_{i}=l \sqrt{N}\right) \geq \frac{2}{\sqrt{2 \pi}} \int_{l}^{\infty} e^{-\frac{s^{2}}{2}} d s-\mathcal{O}\left(N^{-1 / 2}\right) .
$$


Observe that for all $\epsilon>0$ small enough

$$
\frac{\left\lceil t \epsilon^{-1}\right\rceil}{\sqrt{\left\lfloor d \epsilon^{-2}\right\rfloor}} \leq \frac{2 t}{\sqrt{d}} .
$$

Therefore, for all $t<r / 2$ and $\epsilon>0$ small enough, we have

$$
\begin{aligned}
\mathbb{P}\left(\tilde{\tau}_{g} \geq d \epsilon^{-2}\right) & \leq \mathbb{P}\left(\max _{1 \leq m \leq\left\lfloor d \epsilon^{-2}\right\rfloor} \sum_{i=1}^{m} Z_{i}<\left\lceil t \epsilon^{-1}\right\rceil\right) \\
& \leq 1-\frac{2}{\sqrt{2 \pi}} \int_{\frac{2 t}{\sqrt{d}}}^{\infty} e^{-\frac{s^{2}}{2}} d s+\mathcal{O}(\epsilon) .
\end{aligned}
$$

Lemma 3.5 is now an immediate consequence of Lemmas A.2 and A.3.

Next, we prove a technical result (Lemma A.4 below) that we use in Section 3 above. First, in order to keep the calculations simple, let the dimension $n$ be one for now. Assume that $Z$ is distributed according to the uniform distribution in $]-\epsilon, \epsilon$ [ for some $\epsilon>0$. Then for two independent $Z_{1}$ and $Z_{2}$ both distributed as $Z$, the density of the random variable $Z_{1}+Z_{2}$ can be computed via convolution. Thus, since $\left.f_{Z}(x)=1 /(2 \epsilon) \chi\right]-\epsilon, \epsilon[(x)$, we have

$$
\left.f_{Z_{1}+Z_{2}}(x)=\int_{-\infty}^{\infty} f_{Z}(x-y) f_{Z}(y) d y=\left(\frac{1}{2 \epsilon}\right)^{2}(2 \epsilon-|x|) \chi\right]-2 \epsilon, 2 \epsilon[(x) .
$$

For any $k \geq 1$, denote the density $f_{k}:=f_{\sum_{i=1}^{k} Z_{i}}$, where $Z_{i}$ are independent random variables distributed as $Z$. Similarly as in the case $k=2$, we can deduce and prove by induction (see, for example, [17], page 197) that for any $k \geq 1$

$$
f_{k}(x)= \begin{cases}\frac{1}{(k-1) !(2 \epsilon)^{k}} \sum_{j=0}^{\left\lfloor\frac{x+k \epsilon}{2 \epsilon}\right\rfloor}(-1)^{j}\left(\begin{array}{l}
k \\
j
\end{array}\right)(x+k \epsilon-2 j \epsilon)^{k-1}, & \text { if } x \in]-k \epsilon, k \epsilon[, \\
0, & \text { otherwise. }\end{cases}
$$

Unfortunately, it is hard to get quantitative estimates from it.

There have been a lot of studies on the concentration function of a sum of independent random variables (see, for example, [4]). However, we are interested in the pointwise value of the function $f_{k}$ at the origin, and we will estimate the value by hand for the reader in a rather accessible way.

The characteristic function of the random variable $Z$ can be easily calculated,

$$
\varphi_{Z}(t)=\frac{1}{2 \epsilon} \int_{-\epsilon}^{\epsilon} e^{i t x} d x=\frac{\sin (\epsilon t)}{\epsilon t} .
$$

Let $k \geq 2$. Because of the independence,

$$
\varphi_{\sum_{i=1}^{k} Z_{i}}(t)=\left(\frac{\sin (\epsilon t)}{\epsilon t}\right)^{k} .
$$


Now, we have $\int_{-\infty}^{\infty}\left|\varphi_{\sum_{i=1}^{k} Z_{i}}(t)\right| d t<\infty$, so we can use the well-known inversion formula

$$
f_{k}(x)=\frac{1}{2 \pi} \int_{-\infty}^{\infty} e^{-i t x} \varphi_{\sum_{i=1}^{k} Z_{i}}(t) d t .
$$

This inversion formula yields

$$
f_{k}(0)=\frac{1}{\pi} \int_{0}^{\epsilon^{-1}}\left(\frac{\sin (\epsilon t)}{\epsilon t}\right)^{k} d t+\frac{1}{\pi} \int_{\epsilon^{-1}}^{\infty}\left(\frac{\sin (\epsilon t)}{\epsilon t}\right)^{k} d t .
$$

Define

$$
h(z):=2 \frac{1-\cos z}{z^{2}}
$$

so that we have for any $0 \leq m \leq 2 \pi$

$$
\frac{\sin z}{z} \leq 1-h(m) \frac{z^{2}}{6}
$$

for all $|z| \leq m$. This inequality is true since the function $\sin z / z$ decreases for $0<z \leq \pi$ implying

$$
\left(\frac{\sin (m / 2)}{m / 2}\right)^{2} \leq\left(\frac{\sin (z / 2)}{z / 2}\right)^{2}
$$

for all $0<z \leq 2 \pi$. This inequality yields

$$
1-\cos z-h(m) \frac{z^{2}}{2} \geq 0
$$

for all $0<z \leq 2 \pi$ so we have the inequality (A.5), since both sides of the inequality (A.5) are even functions. By using the inequality (A.5), a change of variables formula and the inequality $1-z \leq e^{-z}$ for all $z \in \mathbb{R}$, we have

$$
\begin{aligned}
\frac{1}{\pi} \int_{0}^{\epsilon^{-1}}\left(\frac{\sin (\epsilon t)}{\epsilon t}\right)^{k} d t & =\frac{1}{\pi \epsilon} \int_{0}^{1}\left(\frac{\sin z}{z}\right)^{k} d z \\
& \leq \frac{1}{\pi \epsilon} \int_{0}^{1}\left(1-h(1) \frac{z^{2}}{6}\right)^{k} d z \\
& \leq \frac{1}{\pi \epsilon} \int_{0}^{1} e^{-\frac{z^{2} k h(1)}{6}} d z
\end{aligned}
$$

Again, via changing the variables we derive

$$
\frac{1}{\pi \epsilon} \int_{0}^{1} e^{-\frac{z^{2} k h(1)}{6}} d z \leq \frac{1}{\epsilon} \sqrt{\frac{6}{k \pi h(1)}} \frac{1}{\sqrt{2 \pi}} \int_{0}^{\infty} e^{-\frac{u^{2}}{2}} d u=\sqrt{\frac{3}{2 \pi h(1)}} \frac{1}{\sqrt{k} \epsilon} .
$$


Thus, we have estimated

$$
\frac{1}{\pi} \int_{0}^{\epsilon^{-1}}\left(\frac{\sin (\epsilon t)}{\epsilon t}\right)^{k} d t \leq \sqrt{\frac{3}{2 \pi h(1)}} \frac{1}{\sqrt{k} \epsilon} .
$$

Because $\sin z \leq 1$ for all $z \in \mathbb{R}$, we can estimate the second integral directly, and hence

$$
\frac{1}{\pi} \int_{\epsilon^{-1}}^{\infty}\left(\frac{\sin (\epsilon t)}{\epsilon t}\right)^{k} d t \leq \frac{1}{\pi \epsilon} \int_{1}^{\infty} \frac{1}{z^{k}} d z=\frac{1}{\pi \epsilon(k-1)} .
$$

Therefore, we have derived the estimate

$$
f_{k}(0) \leq \sqrt{\frac{3}{2 \pi h(1)}} \frac{1}{\sqrt{k} \epsilon}+\frac{1}{\pi \epsilon(k-1)} .
$$

Next, we extend the argument to the higher dimensions as well. Assume that $Z$ is a random vector with the uniform distribution in the $n$-ball $B_{\epsilon}(0), n \geq 1$. The density of the random vector $Z$ is

$$
f_{Z}(x)=\frac{1}{\left|B_{\epsilon}(0)\right|} \chi_{B_{\epsilon}(0)}(x) .
$$

Using the same approach as in dimension one, we first need the characteristic function of the random vector $Z$. Denote the measure of the unit ball by $\omega_{n}:=\left|B_{1}(0)\right|=\pi^{n / 2} / \Gamma\left(\frac{n}{2}+1\right)$, where the function $\Gamma$ is the usual gamma function. The random variable $Z$ is invariant under rotation, that is, the density function is a constant on every sphere $S_{r}^{n-1}(0):=\left\{x \in \mathbb{R}^{n}:|x|=r\right\}$ for all $r>0$. Hence, by rotating the ball $B_{\epsilon}(0)$, we see that $\varphi_{Z}(u)=\varphi_{Z}((r, 0, \ldots, 0))$ for all $u \in \mathbb{R}^{n}$ such that $|u|=r$. Let $r>0$, and direct computation with a change of variables $x=\epsilon y$ yields

$$
\begin{aligned}
\varphi_{Z}((r, 0, \ldots, 0)) & =\int_{\mathbb{R}^{n}} e^{i r x_{1}} f_{Z}(x) d x \\
& =\frac{1}{\omega_{n}} \int_{B_{1}(0)} e^{i \varepsilon r y_{1}} d y_{1} \cdots d y_{n} \\
& =\frac{\omega_{n-1}}{\omega_{n}} \int_{-1}^{1}\left(1-y_{1}^{2}\right)^{(n-1) / 2} e^{i \varepsilon r y_{1}} d y_{1} \\
& =\frac{\omega_{n-1}}{\omega_{n}} \int_{-1}^{1}\left(1-y_{1}^{2}\right)^{(n-1) / 2} \cos \left(\varepsilon r y_{1}\right) d y_{1} .
\end{aligned}
$$

A spherical Bessel function of order $n / 2$, often denoted by $J_{n / 2}(z)$, has an integral representation

$$
J_{n / 2}(z)=\left(\frac{z}{2}\right)^{n / 2} \frac{1}{\Gamma\left(\frac{n+1}{2}\right) \sqrt{\pi}} \int_{-1}^{1}\left(1-t^{2}\right)^{\frac{n-1}{2}} \cos (z t) d t
$$


(see, for example, [20]). We can use this integral formula to obtain

$$
\begin{aligned}
& \frac{\omega_{n-1}}{\omega_{n}} \int_{-1}^{1}\left(1-y_{1}^{2}\right)^{(n-1) / 2} \cos \left(\varepsilon r y_{1}\right) d y_{1} \\
& =(\varepsilon r / 2)^{-n / 2} \Gamma\left(\frac{n}{2}+1\right) J_{n / 2}(\varepsilon r) .
\end{aligned}
$$

Thus, we have derived the characteristic function

$$
\varphi_{Z}(u)=\left(\frac{2}{\varepsilon|u|}\right)^{n / 2} \Gamma\left(\frac{n}{2}+1\right) J_{n / 2}(\varepsilon|u|)
$$

for all $u \in \mathbb{R}^{n}$. Spherical Bessel functions have a connection to our calculations in dimension $n=1$, since one could show that

$$
\frac{\sin z}{z}=\sqrt{\frac{\pi}{2 z}} J_{\frac{1}{2}}(z)
$$

holds for all $z \in \mathbb{R}$.

It is possible to express $J_{n / 2}(z)$ as a product of factors such that each factor vanishes at one of the zeros of $z^{-n / 2} J_{n / 2}(z)$. Denote the zeros of the function $z^{-n / 2} J_{n / 2}(z)$ by $\pm j_{n / 2,1}, \pm j_{n / 2,2}, \pm j_{n / 2,3}, \ldots$ with $j_{n / 2, l}>0$ for all $l=1,2, \ldots$ and $j_{n / 2,1} \leq j_{n / 2,2} \leq j_{n / 2,3} \leq$ $\cdots$. Then, we have the infinite product formula of the Bessel function

$$
J_{n / 2}(z)=\left(\frac{z}{2}\right)^{n / 2} \frac{1}{\Gamma\left(\frac{n}{2}+1\right)} \prod_{l=1}^{\infty}\left(1-\frac{z^{2}}{j_{n / 2, l}^{2}}\right)
$$

(see [20], pages 497-498). The number of zeros of $z^{-n / 2} J_{n / 2}(z)$ between the origin and the point

$$
l_{m}:=m \pi+\frac{\pi}{4}(n+1)
$$

is exactly $m$ for all $m$ big enough (see [20], pages 495-497). Consequently, the infinite sum $\sum_{l=1}^{\infty} j_{n / 2, l}^{-2}$ converges, since

$$
\sum_{l=p}^{\infty} j_{n / 2, l}^{-2} \leq \sum_{l=p}^{\infty}\left(\frac{1}{(l-1) \pi+\pi / 4(n+1)}\right)^{2}<\infty
$$

for some $p$ big enough. Therefore, the infinite product in the formula (A.7) is well defined for all $z \in \mathbb{R}$.

Via independence we have

$$
\varphi_{\sum_{i=1}^{k} Z_{i}}(u)=\left(\varphi_{Z}(u)\right)^{k}
$$


and the inversion formula together with the characteristic function (A.6), the infinite product formula (A.7) and a change of variables $z=\epsilon u$ yield

$$
\begin{aligned}
f_{k}(0)= & \frac{1}{(2 \pi)^{n}} \int_{\mathbb{R}^{n}}\left(\varphi_{Z}(u)\right)^{k} d u \\
= & \frac{1}{(2 \pi)^{n} \epsilon^{n}} \int_{B_{s}(0)}\left[\prod_{l=1}^{\infty}\left(1-\frac{|z|^{2}}{j_{n / 2, l}^{2}}\right)\right]^{k} d z \\
& +\frac{2^{k n / 2} \Gamma\left(\frac{n}{2}+1\right)^{k}}{(2 \pi)^{n} \epsilon^{n}} \int_{\mathbb{R}^{n} \backslash B_{s}(0)}\left(\frac{1}{|z|}\right)^{k n / 2}\left(J_{n / 2}(|z|)\right)^{k} d z
\end{aligned}
$$

for all $s>0$.

Now, the function

$$
1-\frac{|z|^{2}}{j_{n / 2, l}^{2}} \geq 0
$$

for all $l \geq 1$, if $0 \leq|z| \leq j_{n / 2,1}$. In addition, since $1-z \leq e^{-z}$ for all $z \in \mathbb{R}$, we have

$$
\begin{aligned}
& \frac{1}{(2 \pi)^{n} \epsilon^{n}} \int_{B_{j_{n / 2,1}}(0)}\left[\prod_{l=1}^{\infty}\left(1-\frac{|z|^{2}}{j_{n / 2, l}^{2}}\right)\right]^{k} d z \\
& \leq \frac{\left|S_{1}^{n-1}\right|}{(2 \pi)^{n} \epsilon^{n}} \int_{0}^{j_{n / 2,1}} e^{-r^{2} k \sum_{l=1}^{\infty} j_{n / 2, l}^{-2} r^{n-1}} d r \\
& \leq \frac{\left|S_{1}^{n-1}\right|}{(2 \pi)^{n} \epsilon^{n}} \int_{0}^{\infty} e^{-r^{2} k \sum_{l=1}^{\infty} j_{n / 2, l}^{-2} r^{n-1}} d r .
\end{aligned}
$$

Hence, we can integrate with a change of variables $r=\left(k \sum_{l=1}^{\infty} j_{n / 2, l}^{-2}\right)^{-1 / 2} t$ to obtain

$$
\begin{aligned}
& \frac{\left|S_{1}^{n-1}\right|}{(2 \pi)^{n} \epsilon^{n}} \int_{0}^{\infty} e^{-r^{2} k \sum_{l=1}^{\infty} j_{n / 2, l}^{-2} r^{n-1}} d r \\
& \quad=\frac{n \int_{0}^{\infty} e^{-t^{2}} t^{n-1} d t}{\Gamma\left(\frac{n}{2}+1\right) \pi^{n / 2} 2^{n}\left(\sum_{l=1}^{\infty} j_{n / 2, l}^{-2}\right)^{n / 2}}\left(\frac{1}{\sqrt{k} \epsilon}\right)^{n} .
\end{aligned}
$$

Thus, there is a constant

$$
c_{n}^{1}:=\frac{n \int_{0}^{\infty} e^{-t^{2}} t^{n-1} d t}{\Gamma\left(\frac{n}{2}+1\right) \pi^{n / 2} 2^{n}\left(\sum_{l=1}^{\infty} j_{n / 2, l}^{-2}\right)^{n / 2}}>0
$$


such that

$$
\frac{1}{(2 \pi)^{n} \epsilon^{n}} \int_{B_{j_{n} / 2,1}(0)}\left[\prod_{l=1}^{\infty}\left(1-\frac{|z|^{2}}{j_{n / 2, l}^{2}}\right)\right]^{k} d z \leq c_{n}^{1}\left(\frac{1}{\sqrt{k} \epsilon}\right)^{n} .
$$

It holds $J_{n / 2}(|z|) \leq 1$ for all $z \in \mathbb{R}^{n}$ (see, for example, [20]). Therefore, we get by a direct calculus

$$
\begin{aligned}
& \frac{2^{k n / 2} \Gamma\left(\frac{n}{2}+1\right)^{k}}{(2 \pi)^{n} \epsilon^{n}} \int_{\mathbb{R}^{n} \backslash B_{j_{n / 2,1}}(0)}\left(\frac{1}{|z|}\right)^{k n / 2}\left(J_{n / 2}(|z|)\right)^{k} d z \\
& \leq \frac{\left|S_{1}^{n-1}\right| 2^{k n / 2} \Gamma\left(\frac{n}{2}+1\right)^{k}}{(2 \pi)^{n} \epsilon^{n}} \int_{j_{n / 2,1}}^{\infty} r^{n-1-k n / 2} d r \\
& =\frac{\left(j_{n / 2,1}\right)^{n}}{2^{n-1} \Gamma\left(\frac{n}{2}+1\right) \pi^{n / 2} \epsilon^{n}}\left(\frac{1}{k-2}\right)\left(\left(\frac{2}{j_{n / 2,1}}\right)^{n / 2} \Gamma\left(\frac{n}{2}+1\right)\right)^{k}
\end{aligned}
$$

for all $k>2$. There exists the following lower bound for the first zero $j_{v, 1}$ (see [5] and for example [3])

$$
j_{v, 1}>v+\frac{\pi+1}{2}>v+2
$$

for all $v>-\frac{1}{2}$. Thus, if $n$ is even, $n=2 h$ for some $h \geq 1$, we get

$$
\frac{\Gamma(h+1) 2^{h}}{\left(j_{h, 1}\right)^{h}}<\frac{h ! 2^{h}}{(h+2)^{h}}<1 .
$$

Similarly, if $n$ is odd, $n=2 h+1$ for some $h \geq 0$, we get

$$
\frac{\Gamma\left(h+\frac{3}{2}\right) 2^{h+1 / 2}}{\left(j_{h+1 / 2,1}\right)^{h+1 / 2}}<\frac{(2 h+2) ! 2^{h} \sqrt{2 \pi}}{4^{h+1}(h+1) !(h+2.5)^{h+1 / 2}}<1 .
$$

Hence, there exists a constant $k_{0}:=k_{0, n}>2$ such that

$$
\left(\frac{1}{k-2}\right)\left(\left(\frac{2}{j_{n / 2,1}}\right)^{n / 2} \Gamma\left(\frac{n}{2}+1\right)\right)^{k} \leq\left(\frac{1}{\sqrt{k}}\right)^{n}
$$

for all $k \geq k_{0}$. Denote

$$
c_{n}^{2}:=\frac{\left(j_{n / 2,1}\right)^{n}}{2^{n-1} \Gamma\left(\frac{n}{2}+1\right) \pi^{n / 2}}>0
$$

and

$$
C_{n}:=2 \max \left\{c_{n}^{1}, c_{n}^{2}\right\}
$$


Thus, we have derived the estimate

$$
f_{k}(0) \leq C_{n}\left(\frac{1}{\sqrt{k} \epsilon}\right)^{n}
$$

for all $k \geq k_{0}$.

Let $k \geq k_{0}$. Theorem A.1 implies that there is a constant $C_{1}:=C_{1, n}>0$ big enough such that for all $\epsilon>0$

$$
\mathbb{P}\left(\left|\sum_{i=1}^{k} Z_{i}\right|<C_{1} \sqrt{k} \epsilon\right) \geq 0.99 .
$$

By using the convolution formula, we have that

$$
\begin{aligned}
f_{k}(x) & =\int_{\mathbb{R}^{n}} f_{k-1}(x-y) \chi_{B_{\epsilon}(0)}(y) d y=\int_{B_{\epsilon}(0)} f_{k-1}(x-y) d y \\
& =\int_{B_{\epsilon}(x)} f_{k-1}(y) d y
\end{aligned}
$$

holds for all $x \in \mathbb{R}^{n}$. The function $f_{1}$ is a decreasing radial function. Thus, we can deduce by using the formula (A.9) that $f_{2}$ is also a decreasing radial function, and by induction $f_{k}$ as well. Therefore, we can denote the density $f_{k}$ as a function of the radius $|u|$ for all $u \in \mathbb{R}^{n}$, and we have for any $\left.C_{*} \in\right] 0, C_{1}[$

$$
f_{k}(0)\left|B_{C_{*} \sqrt{k} \epsilon}(0)\right|+f_{k}\left(C_{*} \sqrt{k} \epsilon\right)\left(\left|B_{C_{1} \sqrt{k} \epsilon}(0)\right|-\left|B_{C_{*} \sqrt{k} \epsilon}(0)\right|\right) \geq 0.99 .
$$

This inequality yields

$$
\begin{aligned}
f_{k}\left(C_{*} \sqrt{k} \epsilon\right) & \geq \frac{0.99-f_{k}(0)\left|B_{C_{*} \sqrt{k} \epsilon}(0)\right|}{\left|B_{C_{1} \sqrt{k} \epsilon}(0)\right|-\left|B_{C_{*} \sqrt{k} \epsilon}(0)\right|} \\
& \geq \frac{0.99}{\left|B_{C_{1} \sqrt{k} \epsilon}(0)\right|}-f_{k}(0)\left(\frac{C_{*}}{C_{1}}\right)^{n} .
\end{aligned}
$$

Now, we use the estimate (A.8) to obtain

$$
\begin{aligned}
f_{k}\left(C_{*} \sqrt{k} \epsilon\right) & \geq \frac{0.99}{\left|B_{C_{1} \sqrt{k} \epsilon}(0)\right|}-C_{n}\left(\frac{C_{*}}{C_{1} \sqrt{k} \epsilon}\right)^{n} \\
& =\left(\frac{1}{C_{1}}\right)^{n}\left(\frac{0.99}{\omega_{n}}-C_{n}\left(C_{*}\right)^{n}\right)\left(\frac{1}{\sqrt{k} \epsilon}\right)^{n} .
\end{aligned}
$$

Thus, we have proven the following lemma.

Lemma A.4. Let $\epsilon>0$ and let $Z$ be distributed according to the uniform distribution in the ball $B_{\epsilon}(0) \subset \mathbb{R}^{n}$. For any $k \geq 2$, denote the density of the random variable $\sum_{i=1}^{k} Z_{i}$ by $f_{k}$, where 
the random variables $Z_{i}, i \in\{1, \ldots, k\}$, are independent and distributed as $Z$. Then $f_{k}$ is a decreasing radial function, and there exist universal constants $k_{0}:=k_{0, n}>2, C_{1}:=C_{1, n}>0$ and $C_{n}>0$ such that for all $k \geq k_{0}$ and $\left.C_{*} \in\right] 0, C_{1}[$ we have

$$
f_{k}\left(C_{*} \sqrt{k} \epsilon\right) \geq\left(\frac{1}{C_{1}}\right)^{n}\left(\frac{0.99}{\omega_{n}}-C_{n}\left(C_{*}\right)^{n}\right)\left(\frac{1}{\sqrt{k} \epsilon}\right)^{n} .
$$

Observe that

$$
f_{k}\left(C_{*} \sqrt{k} \epsilon\right) \geq \zeta\left(\frac{1}{\sqrt{k} \epsilon}\right)^{n}
$$

for some $\zeta:=\zeta_{n}>0$, if we choose $C_{*}>0$ so small that

$$
C_{*}<\left(\frac{0.99}{\omega_{n} C_{n}}\right)^{1 / n} .
$$

\section{References}

[1] Armstrong, S.N. and Smart, C.K. (2010). An easy proof of Jensen's theorem on the uniqueness of infinity harmonic functions. Calc. Var. Partial Differential Equations 37 381-384. MR2592977

[2] Atar, R. and Budhiraja, A. (2010). A stochastic differential game for the inhomogeneous $\infty$-Laplace equation. Ann. Probab. 38 498-531. MR2642884

[3] Elbert, Á. (2001). Some recent results on the zeros of Bessel functions and orthogonal polynomials. In Proceedings of the Fifth International Symposium on Orthogonal Polynomials, Special Functions and Their Applications (Patras, 1999) 133 65-83. MR1858270

[4] Esseen, C.G. (1968). On the concentration function of a sum of independent random variables. Z. Wahrsch. Verw. Gebiete 9 290-308. MR0231419

[5] Ifantis, E.K. and Siafarikas, P.D. (1985). A differential equation for the zeros of Bessel functions. Appl. Anal. 20 269-281. MR0814954

[6] Kawohl, B., Manfredi, J. and Parviainen, M. (2012). Solutions of nonlinear PDEs in the sense of averages. J. Math. Pures Appl. (9) 97 173-188. MR2875296

[7] Klenke, A. (2008). Probability Theory: A Comprehensive Course. Universitext. London: Springer. MR2372119

[8] Krylov, N.V. and Safonov, M.V. (1979). An estimate for the probability of a diffusion process hitting a set of positive measure. Dokl. Akad. Nauk SSSR 245 18-20. MR0525227

[9] Krylov, N.V. and Safonov, M.V. (1980). A property of the solutions of parabolic equations with measurable coefficients. Izv. Akad. Nauk SSSR Ser. Mat. 44 161-175, 239. MR0563790

[10] Luiro, H., Parviainen, M. and Saksman, E. (2013). Harnack's inequality for $p$-harmonic functions via stochastic games. Comm. Partial Differential Equations 38 1985-2003. MR3169768

[11] Luiro, H., Parviainen, M. and Saksman, E. (2014). On the existence and uniqueness of $p$-harmonious functions. Differential Integral Equations 27 201-216. MR3161602

[12] Manfredi, J.J., Parviainen, M. and Rossi, J.D. (2010). An asymptotic mean value characterization for p-harmonic functions. Proc. Amer. Math. Soc. 138 881-889. MR2566554

[13] Manfredi, J.J., Parviainen, M. and Rossi, J.D. (2012). On the definition and properties of p-harmonious functions. Ann. Sc. Norm. Super. Pisa Cl. Sci. (5) 11 215-241. MR3011990 
[14] Oberman, A.M. (2005). A convergent difference scheme for the infinity Laplacian: Construction of absolutely minimizing Lipschitz extensions. Math. Comp. 74 1217-1230 (electronic). MR2137000

[15] Peres, Y., Schramm, O., Sheffield, S. and Wilson, D.B. (2009). Tug-of-war and the infinity Laplacian. J. Amer. Math. Soc. 22 167-210. MR2449057

[16] Peres, Y. and Sheffield, S. (2008). Tug-of-war with noise: A game-theoretic view of the $p$-Laplacian. Duke Math. J. 145 91-120. MR2451291

[17] Rényi, A. (1970). Probability Theory. Amsterdam: North-Holland. MR0315747

[18] Ruosteenoja, E. (2016). Local regularity results for value functions of tug-of-war with noise and running payoff. Adv. Calc. Var. 9 1-17. MR3441079

[19] Shiryaev, A.N. (1996). Probability, 2nd ed. Graduate Texts in Mathematics 95. New York: Springer. MR1368405

[20] Watson, G.N. (1944). A Treatise on the Theory of Bessel Functions. Cambridge: Cambridge Univ. Press. MR0010746

Received October 2015 and revised March 2016 